\title{
Role of Somatosensory Cortex in Visuospatial Attention
}

\author{
Daniela Balslev, ${ }^{1,2}$ Bartholomäus Odoj, ${ }^{1,2}$ and Hans-Otto Karnath ${ }^{1,3}$ \\ ${ }^{1}$ Center of Neurology, Division of Neuropsychology, Hertie Institute for Clinical Brain Research, University of Tübingen, D-72076 Tübingen, Germany, \\ ${ }^{2}$ Department of Psychology, University of Copenhagen, DK-1353 Copenhagen, Denmark, and ${ }^{3}$ Department of Psychology, University of South Carolina, \\ Columbia, South Carolina 29208
}

The human somatosensory cortex (S1) is not among the brain areas usually associated with visuospatial attention. However, such a function can be presumed, given the recently identified eye proprioceptive input to S1 and the established links between gaze and attention. Here we investigated a rare patient with a focal lesion of the right postcentral gyrus that interferes with the processing of eye proprioception without affecting the ability to locate visual objects relative to her body or to execute eye movements. As a behavioral measure of spatial attention, we recorded fixation time during visual search and reaction time for visual discrimination in lateral displays. In contrast to a group of age-matched controls, the patient showed a gradient in looking time and in visual sensitivity toward the midline. Because an attention bias in the opposite direction, toward the ipsilesional space, occurs in patients with spatial neglect, in a second study, we asked whether the incidental coinjury of S1 together with the neglect-typical perisylvian lesion leads to a milder neglect. A voxelwise lesion behavior mapping analysis of a group of right-hemisphere stroke patients supported this hypothesis. The effect of an isolated S1 lesion on visual exploration and visual sensitivity as well as the modulatory role of $S 1$ in spatial neglect suggest a role of this area in visuospatial attention. We hypothesize that the proprioceptive gaze signal in S1, although playing only a minor role in locating visual objects relative to the body, affects the allocation of attention in the visual space.

\section{Introduction}

Visuospatial attention can be defined as the selection of a location in the visual space for preferential stimulus processing. Previous research has associated this function with a network in the higher-order frontal, parietal, and temporal cortices and subcortical structures, such as thalamus and superior colliculi (Corbetta and Shulman, 2002; Bisley and Goldberg, 2010; Karnath and Rorden, 2012; Petersen and Posner, 2012). The primary somatosensory cortex (S1) is not among these areas. However, a role of $\mathrm{S} 1$ in visuospatial attention can be presumed, given its recently discovered proprioceptive gaze input (Wang et al., 2007; Balslev and Miall, 2008) and the established links between gaze and attention. First, many oculomotor areas, such as the superior colliculi or the frontal eye fields, have an additional function in spatial attention. For instance the coinjury of the superior colliculus (Sprague, 1966; Weddell, 2004) or the frontal eye field (Vuilleumier et al., 1996) can alleviate spatial neglect. Second, to stabilize the focus of attention across eye movements or to allow cross-modal interactions in spatial attention, locations must be coded in non-retinotopic coordinates (Andersen et al., 1985;

\footnotetext{
Received March 14, 2013; revised 0ct. 7, 2013; accepted 0ct. 12, 2013.

Author contributions: D.B. and H.-O.K. designed research; D.B., B.0., and H.-0.K. performed research; D.B., B.O., and H.-0.K. analyzed data; D.B., B.O., and H.-0.K. wrote the paper.

This work was supported by Danish Medical Research Councils Grant 09-072209 (D.B.), a Marie Curie IntraEuropean Fellowship for Career Development within the Seventh European Community Framework Programme (D.B.), and Deutsche Forschungsgemeinschaft Grant KA 1258/10-1 (H.-0.K.). The authors are grateful to RW for helping us conduct this study.

The authors declare no competing financial interests.

Correspondence should be addressed to Daniela Balslev, School of Psychology and Neuroscience, University of St. Andrews, St. Mary's Quad, South Street, St. Andrews, KY16 9JP, UK. E-mail: d.balslev@gmail.com.

DOI:10.1523/JNEUROSCI.1112-13.2013

Copyright $\odot 2013$ the authors $\quad 0270-6474 / 13 / 3318311-08 \$ 15.00 / 0$
}

Galletti et al., 1993) or updated across eye movements (Duhamel et al., 1992). These processes require gaze information. Eye proprioception, as well as the efference copy of the oculomotor command, provide such information and could thus be used for coding the locus of attention.

To investigate whether S1 plays a role in visuospatial attention, here we conducted two studies in brain-lesioned patients. Study 1 examined a patient with a rare circumscribed stroke in the right postcentral gyrus. This patient has a deficit in processing proprioceptive input from the left, contralesional, eye. Her ability to locate visual objects relative to the body was normal, presumably by relying exclusively on the efference copy of the motor command (Balslev et al., 2012a). We recorded the patient's fixation time during visual search in lateral, left and right, letter arrays (Experiment A) as well as the reaction time for discriminating left- and right-hemifield targets presented at equal retinal eccentricity in lateral left and right displays (Experiment B). The setup with lateral visual displays was guided by our previous work. After inhibitory repetitive transcranial magnetic stimulation (rTMS) over S1, both perceived gaze and the locus of attention shift toward the midline, that is to the left in rightward gaze and to the right in leftward gaze (Odoj and Balslev, 2013). Therefore, we hypothesized that an isolated S1 stroke would cause a bias in visual search that depends on the direction of gaze (Study 1). Study 2 asked whether the coinjury of S1 exerts a modulatory effect on the ipsilesional selection bias in spatial neglect. Patients with spatial neglect, usually after a right perisylvian lesion (Karnath and Rorden, 2012), show a lateral bias during visual search (Hornak, 1992; Karnath, 1994; Karnath et al., 1998). The prediction of a modulatory effect of an S1 lesion on this lateral bias was tested using voxelwise symptom lesion 
mapping (VLBM) in a group of patients with large right-hemisphere strokes.

\section{Materials and Methods \\ Study 1: spatial attention in a patient with a focal S1 lesion \\ Participants}

We investigated patient RW, a 69-year-old right-handed woman. Her vision was corrected to normal with glasses. RW had a focal intracerebral hemorrhage with a length of 3.6 $\mathrm{cm}$ centered on the right postcentral gyrus (Fig. 1). The lesion overlapped Brodmann area (BA) 3a, BA 2, and BA 1 as defined by the Juelich atlas (Geyer et al., 1996; Grefkes et al., 2001; Eickhoff et al., 2005). On this atlas, it was possible to localize $94.6 \%$ of the lesion volume, $80 \%$ of it being in S1 (39.9\% in area 2, $19.7 \%$ in area $3 b, 15.9 \%$ in area 1 , and $4.5 \%$ in area $3 a)$. Less than $4 \%$ of the voxels were also found in area $7(3.9 \%)$, area 4 posterior $(3.2 \%)$, inferior parietal cortex (IPC) $(2.9 \%)$, and area 4 anterior $(2.8 \%)$. Her lesion overlapped with the projection of eye muscle proprioception in the sensorimotor cortex identified in healthy subjects (Balslev et al., 2011a). At the time of testing, $\sim 3$ years after stroke, RW had a proprioceptive deficit in the contralesional left eye (Balslev et al., 2012a). We found that, in normal conditions, the patient perceived her direction of gaze as accurately and precisely as the control group, presumably by relying on the efference copy of the oculomotor command. However, in special circumstances, when a mismatch between the efference copy of the oculomotor command and eye proprioception prompted the use of the erroneous eye proprioceptive information (i.e., a brief and gentle eye push applied to the closed eye), the patient underestimated her angle of gaze. Namely, after a brief push to the eyeball, she mislocated an LED at $\sim 15^{\circ}$ laterally as being in front of her nose (Balslev et al., 2012a).

Neuropsychological assessments showed no neglect, no extinction, and no optic ataxia. The patient had normal, symmetric sensitivity to touch as assessed by two-point discrimination on the hands (Borchers et al., 2011) and face (Balslev et al., 2012a). As controls, we tested 11 healthy participants in Experiment A (10 women; mean age, 64.1 years; range, 59-74 years) and 14 healthy participants in Experiment B (11 women; mean age, 66.7 years; range, 60-74 years). The mean age in control groups was not significantly different from the patient's age [ $t$ test, singlecase comparison (Crawford and Howell, 1998), both $p>0.35$ ]. All controls were right-handed and had normal or corrected-to-normal vision with glasses. All experiments were approved by the Ethics Committee at the University Clinic of Tübingen.

\section{Procedure}

Experiment $A$. This experiment investigated whether a lesion of the right postcentral gyrus affects the spatial distribution of fixations during visual search. The task was adapted from Karnath et al. (1998). The experiment was conducted in monocular vision. Although proprioceptive information from both eyes is relayed to each brain hemisphere (Balslev et al., $2011 b$ ), the input from the contralateral eye is functionally more important (Balslev et al., 2012a). The non-viewing eye was covered with a patch. The participants were instructed to look for a single, non-existent target (letter A) in a random field of letters while their gaze position was recorded. The letter arrays were placed laterally, to the left or right of the participants' midline. The head was fixed using a chin rest and cheek pads. They viewed a $25^{\circ}$-wide letter array $\left(50^{\circ}\right.$ height) centered at $14^{\circ}$ visual angle to the left or to the right of the orbit midline of the uncovered eye (Fig. 2A,C). The array showed randomly distributed black capital letters from $B$ to $Z\left(1^{\circ}\right.$ visual angle, 113 letters in the left array and 119 letters in the right array). Each letter was printed on a separate piece of white paper, attached to the display. Unbeknownst to the participants, the letter A was absent from the arrays, so the participants had to keep searching throughout the trial. Before each trial, the arrays were covered. At the beginning of the trial, the cover was removed and the participants were instructed to search one lateral array. Eye movements were re-

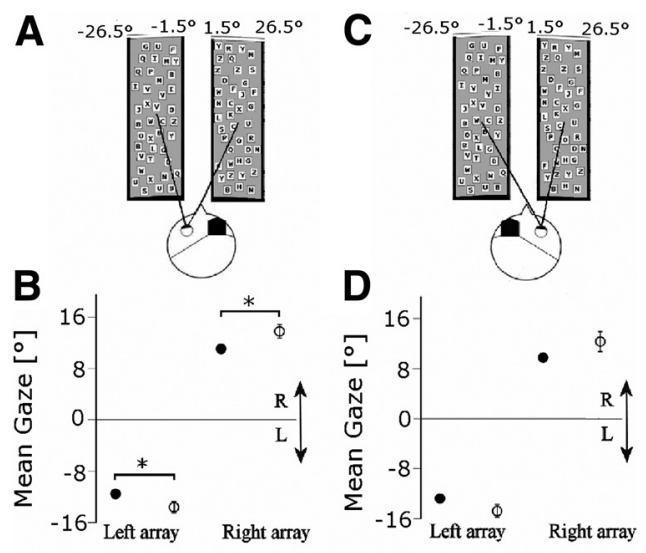

Figure 2. Mean gaze during visual search after a focal, right $\mathrm{S} 1$ lesion. Participants searched for a (nonexistent) target among random letters. The center of the letter array was at $14^{\circ}$, to the left or right of the orbit center. Mean gaze for the patient (filled circles) and a group of 11 healthy, age-matched, controls (open circles) are shown for the left (contralesional, $\boldsymbol{A}, \boldsymbol{B}$ ) and the right (ipsilesional, $\boldsymbol{C}, \boldsymbol{D}$ ) eye. Error bars represent the $95 \%$ confidence interval. Negative values on the $y$-axis describe leftward gaze, and positive values describe rightward gaze. Zero corresponds to the position of the midline of the orbit on a horizontal line. ${ }^{*} p<0.05$ for a single-case $t$ test for a difference between patient RW and the control group (Crawford and Howell, 1998).

corded for $20 \mathrm{~s}$, and then the array was again covered. Each participant completed four trials of $20 \mathrm{~s}$ duration, one for each lateral array and eye. The order of conditions was left eye-leftward array, left eye-rightward array, right eye-leftward array, and right eye-rightward array. To keep the participants motivated, in a practice trial before data registration, a letter A was attached at the center of the left array. If the participant did not find the target within $20 \mathrm{~s}$, the experimenter identified its location by pointing to it.

Eye position was recorded with a head-mounted tracker (EYELINK II; SR Research) that sampled pupil location at $250 \mathrm{~Hz}$. The tracker was calibrated for each lateral position of the array using a $3 \times 3$ grid. Blinks and saccades were detected using the SR EyeLink algorithm. Saccades were defined by an amplitude of at least $0.5^{\circ}$, using an acceleration threshold of $9500 \% \mathrm{~s}^{2}$ and a velocity threshold of $30 \%$ s. After excluding blinks, we calculated mean eye position during visual search. To investigate whether changes in mean eye position were caused by changes in the fixation time or in the amplitude/frequency of the saccades, these parameters were calculated too and compared across conditions using the modified $t$ test for the single-case comparison (Crawford and Howell, 1998).

Experiment B. This experiment investigated whether a lesion of the right postcentral gyrus causes a lateral asymmetry in visual sensitivity. The task, similar to that used previously (Odoj and Balslev, 2013), was performed in left (contralesional) monocular vision while the right eye was covered with a patch. The choice of the left eye was motivated by the 


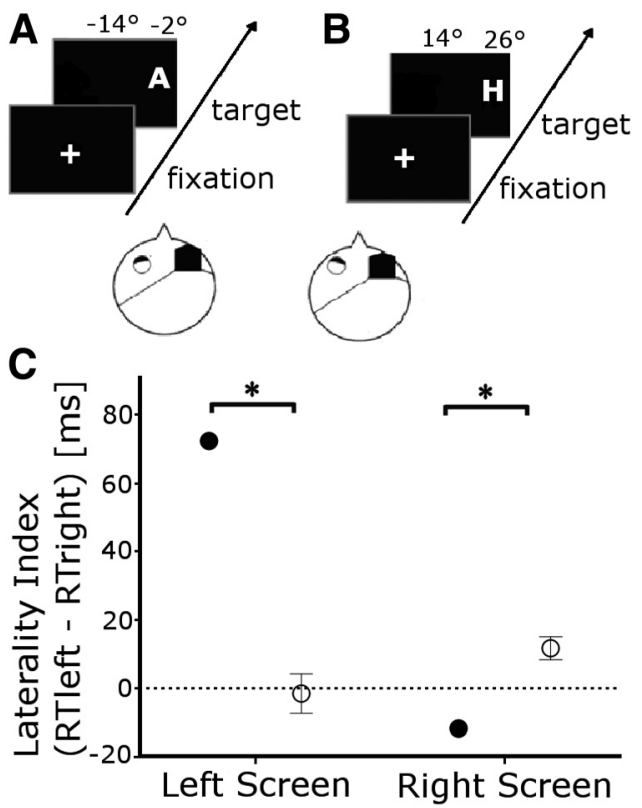

Figure 3. Increased visual sensitivity at locations nearer the midline after a right $\mathrm{S} 1$ lesion. In left eye vision, participants fixated at $14^{\circ}$ to the left $(\boldsymbol{A})$ or right $(\boldsymbol{B})$ from the center of the left orbit. A target letter, $\mathrm{A}$ or $\mathrm{H}$, was presented at $12^{\circ}$, to either the left or right of fixation with equal probability. The laterality index ( $\left(\mathrm{RT}_{\text {left }}-\mathrm{RT}_{\text {right }}\right.$ ) showed a significant interaction between group and gaze direction (C). Filled circles indicate the patient, and open circles indicate controls ( $n=14$, mean \pm SE). ${ }^{*} p<0.05$, one-tailed single-case $t$ test (Crawford and Howell, 1998).

results of Experiment A, as well as by the stronger impairment in proprioceptive inflow in the contralesional versus ipsilesional eye in RW (Balslev et al., 2012a). The head was fixed in a chin rest using cheek pads. A computer screen was positioned with its center at $14^{\circ}$ to the left or to the right from the orbit midline of the left eye (Fig. $3 A, B$ ). At the start of the trial, a fixation cross $\left(1^{\circ} \times 1^{\circ}\right)$ was presented at the center of the screen for a randomized time interval between 1740 and $2540 \mathrm{~ms}$. Then a target letter $\left(\mathrm{A}\right.$ or $\left.\mathrm{H}, 1^{\circ} \times 1^{\circ}\right)$ appeared for $120 \mathrm{~ms}$ at $12^{\circ}$ visual angle to either the left or right of fixation. The participant was instructed to fixate the cross throughout the trial and to respond as fast as possible whether he or she has seen an A or an H. A response was required for each trial, and if in doubt about the correct response, the participant was instructed to guess. To respond, the participant pressed one of two buttons on a response box using the right hand placed at the body midline. Participants used their index finger to respond $\mathrm{A}$ and middle finger for $\mathrm{H}$. A new trial started immediately after a response. Each participant completed 288 trials, the first 144 with the screen on the left side and the last 144 trials with the screen on the right side. The different types of trials (target left/right, letter $\mathrm{A} / \mathrm{H}$ ) were equiprobable and intermixed in a pseudorandomized order, identical across all participants. Left-right asymmetries in visual sensitivity were indexed by a laterality index calculated as the difference between reaction times $\left(\mathrm{RT}_{\text {left }}-\mathrm{RT}_{\text {right }}\right)$. We compared this index across groups (patient vs controls) and screen conditions (left vs right). Because hand posture and motor response were the same across conditions, a group $\times$ condition interaction in reaction time cannot be attributed to a sensorimotor deficit.

To verify fixation, the position of the left eye was recorded with a head-mounted tracker like in Experiment A. Mean gaze position was calculated for $200 \mathrm{~ms}$ before target onset. Only trials with a gaze deviation of $<1^{\circ}$ from the fixation cross were included in the analysis (RW, $83.7 \%$; control group, $95.7 \pm 6.1 \%$, mean $\pm \mathrm{SD}$ ).

\section{Study 2: VBLM in right-hemisphere stroke patients Participants}

We reanalyzed patient data collected in two previous studies (Becker and Karnath, 2010; Karnath et al., 2011). From this data pool, we selected patients who suffered a first-ever stroke resulting in a right-hemisphere lesion that was predominantly cortical (rather than subcortical) and that covered at least $50 \%$ of the perisylvian network identified previously to be associated with acute neglect (Karnath et al., 2011). To calculate how much of this region of interest was covered by each individual lesion, the statistical map resulting from the VLBM analysis conducted in the study of Karnath et al. (2011) was first converted into a binary map. Then the overlap percentage was calculated as the number of voxels within the region of interest that were lesioned in the individual patient, multiplied by 100 and divided by the total number of voxels of the region of interest.

A total of 15 patients ( 11 females; mean age, 64 years; range, 45-80 years) fulfilled the inclusion criteria. The selected patients had cortical ischemic lesions that covered on average $68.6 \%$ of the region of interest (range, 51.7-93.4\%). Figure 4 shows a simple overlap of the brain lesions in the included patients. In accord with the inclusion criteria, all lesions were centered on the perisylvian region. However, because all lesions extended beyond this neglect-typical region, the figure shows additional areas that were lesioned in some but not all patients. Relevant for the hypothesis of this study, the area of partial overlap was distributed symmetrically around the right perisylvian region and included, among other areas, the right postcentral gyrus. This allowed us to investigate whether any of these areas of partial overlap had an effect on the neglect scores when lesioned compared with when intact.

\section{Imaging and behavioral data}

The mean time from stroke onset to imaging for the selected patients was $1.1 \mathrm{~d}$ (range, $0-5 \mathrm{~d}$ ). In the previous studies (Becker and Karnath, 2010; Karnath et al., 2011), lesions had been delineated using MRIcroN software (Rorden et al., 2007; www.mricro.com). In those patients with spiral computed tomography scanning at admission $(n=9)$, lesions had been drawn directly on the slices of a normalized T1-weighted template MRI scan from the Montreal Neurological Institute (MNI) with a $1 \times 1$ $\mathrm{mm}$ in-plane resolution, distributed with the MRIcroN toolset. In the patients who underwent MRI scanning at admission $(n=6)$, the boundary of the lesion had been delineated directly on the image for every single transverse slice using MRIcroN software. Both the MRI scan and the lesion shape then had been mapped into stereotaxic space using the normalization algorithm provided by SPM5 as well as cost-function masking when calculating transformation parameters (Brett et al., 2001).

The severity of spatial neglect was indexed by the center of cancellation (CoC) measure (Rorden and Karnath, 2010) obtained from the letter cancellation task (Weintraub and Mesulam, 1985), the Bells test (Gauthier et al., 1989), and/or the Albert's test (Albert, 1973). The tests were performed as soon as the patients were able to participate, on average $4.9 \mathrm{~d}$ after stroke (range, $0-19 \mathrm{~d}$ ). When more than one test result was available ( $80 \%$ of cases), the $\mathrm{CoC}$ measures were averaged across tests. The mean \pm SD CoC score for all patients was $0.64 \pm 0.34$. Twelve of these 15 patients had spatial neglect, defined as a $\mathrm{CoC}>0.083$ (Rorden and Karnath, 2010).

\section{Data analysis}

The imaging and behavioral data were entered into a VLBM analysis. The analysis is implemented in MRIcroN using $t$ test statistics and the continuous $\mathrm{CoC}$ score as the dependent variable. This analysis identified voxels whose lesion is correlated with a less severe neglect, i.e., with lower $\mathrm{CoC}$ scores, as well as with a more severe neglect, i.e., higher CoC scores. Only voxels in which at least $20 \%$ of patients $(n=3)$ were assigned to each statistical group were included. This was done to avoid a possible influence from just one or two data outliers. The threshold for statistical analysis was set at 0.05 and corrected for multiple comparisons using false discovery rate (FDR).

\section{Results}

Study 1: spatial attention in a patient with a focal S1 lesion $R W$ fixates longer at locations nearer the midline during visual search

To investigate whether an S1 lesion causes a bias in spatial attention, we recorded gaze direction while the subjects searched in lateral letter arrays. We hypothesized a lateral shift in the spatial 


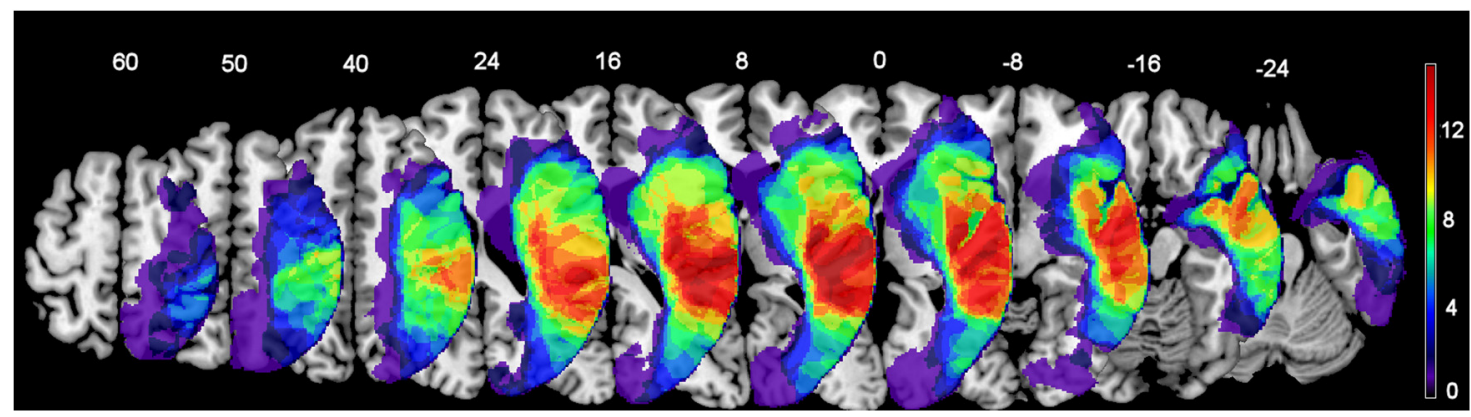

Figure 4. Simple overlap of the individual lesions for the 15 patients included in Study 2. For each voxel, the number of patients with a lesion at that location is color coded. The color map is superposed on a single-subject T1 template coregistered with the MNI152 template. Conventions as in Figure 1.
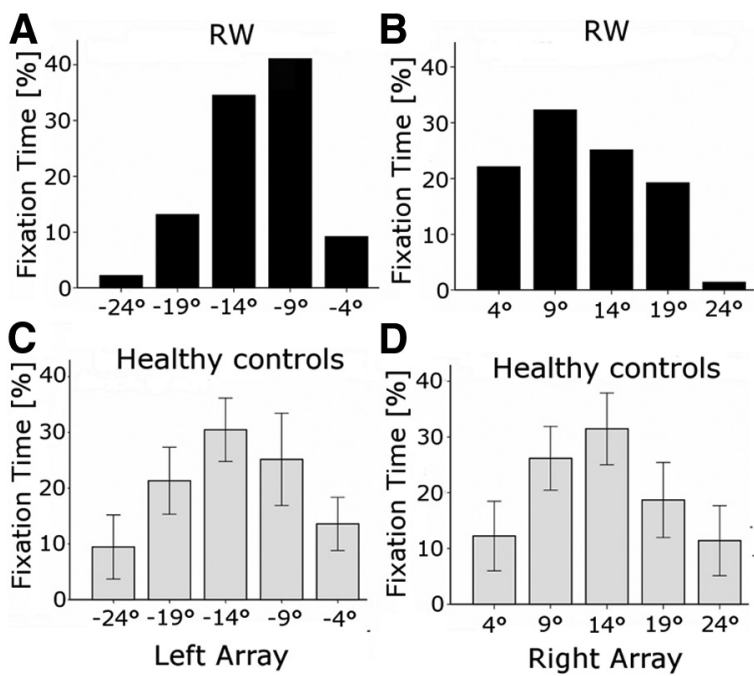

Figure 5. Distribution of the fixation time during visual search after an $\mathrm{S} 1$ lesion. In left (contralesional) eye vision, the patient fixated longer in the inner area of the left $(\boldsymbol{A})$ and right $(\boldsymbol{B})$ arrays, whereas the distribution of the fixation time in the healthy controls $(\boldsymbol{C}, \boldsymbol{D})$ was symmetrical relative to the center of the arrays. The figure shows the percentage of the fixation time spent in each $5^{\circ}$ sector of the arrays along the horizontal axis. For healthy controls $(n=$ $11)$, the bars show mean $\pm 1 S D$. The position of the left orbit midline is at $0^{\circ}$.

distribution of fixation time. In accord with this hypothesis, mean eye position during visual search in RW was $\sim 2^{\circ}$ nearer the midline compared with that of the healthy, age-matched controls. Mean eye position in RW for the left eye was $-11.5^{\circ}$ in the left array and $11.1^{\circ}$ in the right array, whereas in the control group these values were $-13.7 \pm 0.9^{\circ}$ and $13.8 \pm 1.08^{\circ}$, respectively (Fig. $2 B$ ). Mean eye position in RW for the right eye was $-12.7^{\circ}$ in the left array and $9.8^{\circ}$ in the right array. In the control group, the mean eye position was $-14.7 \pm 1.0^{\circ}$ and $12.3 \pm 1.6^{\circ}$ (Fig. 2D). The modified $t$ test for the single-case comparison (Crawford and Howell, 1998) showed a statistically significant difference for the contralesional left eye $(t=2.28, p=0.045$ for left array and $t=-2.42, p=0.035$ for right array). For the ipsilesional right eye, we found a numerical difference in the same direction, which was not statistical significant $(t=1.86, p=0.09$ for left array and $t=-1.55, p=0.15$ for right array).

The shift in mean eye position in RW's left eye was caused by a shift in the spatial distribution of the fixation time (Fig. 5). The patient fixated 55.38\% longer in the inner versus outer half of the left array (control group, $5.27 \pm 19.00 \%$ longer in the inner half) and $37.91 \%$ longer in the inner versus outer half of the right array (control group, $1.43 \pm 14.20 \%$ longer in the inner half). The modified $t$ test for the single-case comparison (Crawford and
Howell, 1998) showed a statistically significant difference between these values $(t=-2.52, p=0.030$ for left array and $t=$ $2.46, p=0.034$ for right array).

Thus, the results of Experiment A show a bias in visual exploration toward the midline after an S1 lesion. This contrasts with the bias in opposite direction, away from midline, found previously after inhibitory rTMS in the same area (Odoj and Balslev, 2013). A notable difference between the two studies is that an acute decrease of the excitability of the eye proprioceptive area with rTMS causes an error in perceived gaze direction toward the midline (Balslev and Miall, 2008; Odoj and Balslev, 2013), whereas a chronic lesion in this area, although it interferes with the processing of the eye proprioceptive input, does not affect the gaze position estimate (Balslev et al., 2012a). Therefore, the apparent contradiction between the two results can be resolved by assuming that the shift in exploratory eye movements away from midline after rTMS in the study by Odoj and Balslev (2013) may have reflected a misperception of the direction of gaze, i.e., the participants may have felt their gaze to be less eccentric than it actually was and therefore searched at more peripheral locations in the array. After a chronic S1 lesion in patient RW, the gaze angle is correctly perceived (Balslev et al., 2012a). This may be the reason why, here, the same spatial bias was observed in both visual sensitivity (Experiment B) and visual exploration (Experiment A).

$R W$ 's bias toward the midline in looking time cannot be explained by a lower-level oculomotor deficit

One could object that the shift in mean eye position and fixation time toward the midline in RW reflects an inability to move the eyes toward peripheral locations or altered saccade metrics. We think that these explanations are unlikely for the following reasons.

First, Experiment A did not find a difference between RW and the control group in the range of eye movements. When viewing the array with the left eye, the patient moved her gaze between $\left[-22.0^{\circ}\right.$ to $\left.-2.7^{\circ}\right]$ for the left and $\left[2.0^{\circ}\right.$ to $\left.22.4^{\circ}\right]$ for the right array. For the left array, controls moved their gaze between $-24.7 \pm 1.7^{\circ}$ and $-2.2 \pm 0.8^{\circ}$ (mean $\pm \mathrm{SD}$ ). For the right array, the movement interval in healthy controls was between $3.1 \pm 1.0^{\circ}$ and $24.5 \pm 2.3^{\circ}$. None of these values were significantly different from the values in the patient (modified $t$ tests for single-case comparison, all $p>0.16$ )

Second, we found no significant difference between RW and the control group in the number of fixations in the left/right half of the letter arrays. All modified $t$ tests for the single-case comparison showed $p>0.24$. Likewise, the analysis of saccade frequency or amplitude showed no significant difference between RW and the control group for any of the four combinations of 


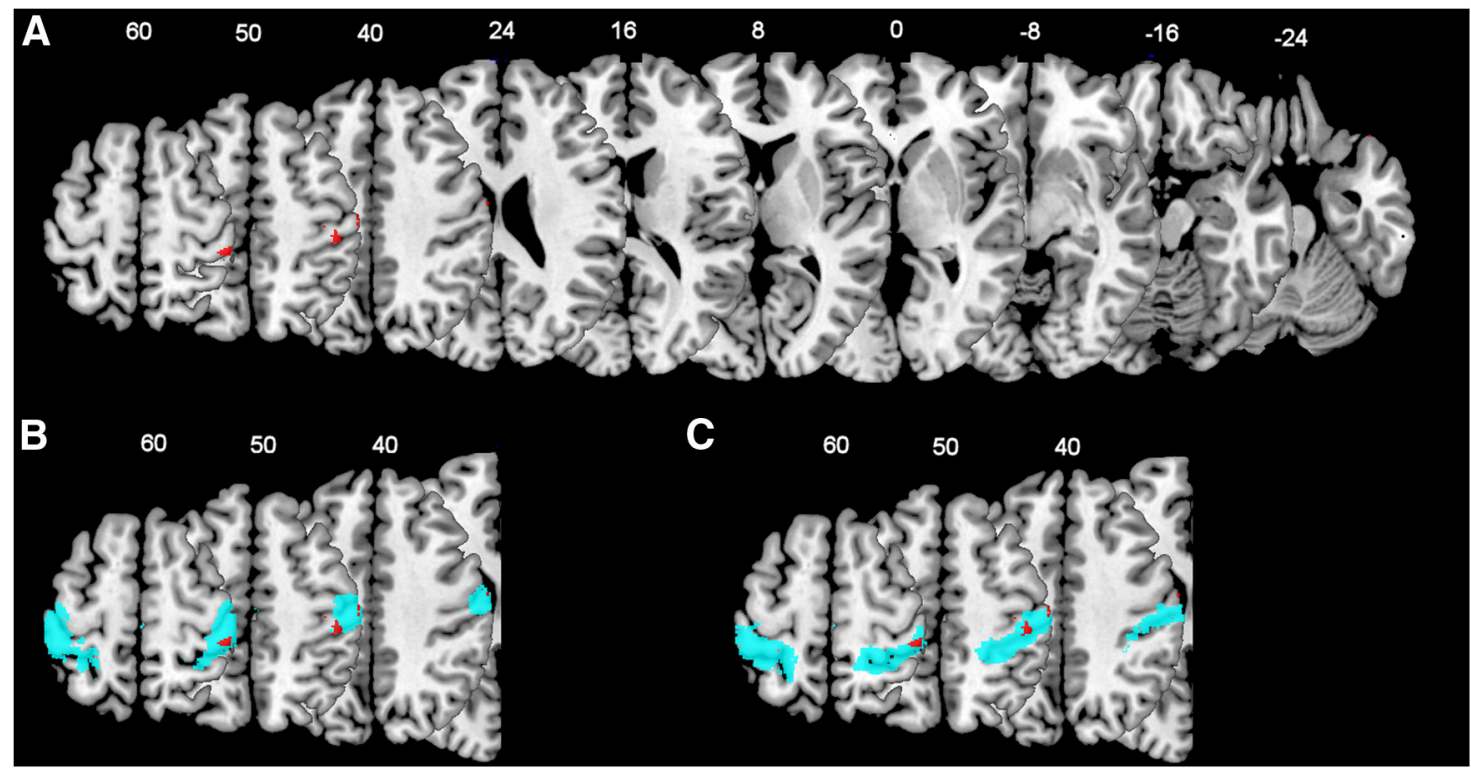

Figure 6. Brain areas in which an additional lesion was associated with milder neglect, given a primary lesion in the perisylvian network region. The statistical map (red) is thresholded at $p<$ 0.05 , FDR corrected (zscore $>2.16$ ) and overlaid on a single-subject T1 template coregistered with the MNI152 template $(\boldsymbol{A})$. The probabilistic maps from the Juelich atlas (Geyer et al., 2000; Grefkes et al., 2001; Eickhoff et al., 2005) for BA 1 (B) and BA 2 (C) thresholded at a voxel probability for the BA $=25 \%$ are shown in blue. Conventions as in Figure 1.

saccade direction (leftward or rightward) and array (left or right). All modified $t$ tests for the single-case comparison showed a $p>$ 0.48 for amplitude and a $p>0.07$ for frequency. There was a numerical tendency for fewer leftward saccades while searching the left array in the patient compared with the controls $(p=$ $0.07)$. For the other combinations of saccade direction, array, and eye, all $p$ values were 0.21 .

\section{$R W$ shows a gradient in visual sensitivity that favors locations} nearer midline

The argument for a role of S1 in visuospatial attention and against a purely ocular sensorimotor explanation for the asymmetry in fixation time in Experiment $A$ is based on a null result. To address this shortcoming, Experiment B investigated spatial attention using a task in which the sensorimotor response was the same in all conditions. Like Experiment A, Experiment B showed a spatial bias toward midline in RW. RW needed longer time to discriminate targets located further versus nearer from midline, and this difference was significantly larger in the patient than in the control group (Fig. $3 C$ ). In leftward gaze, the laterality index for reaction time $\left(\mathrm{RT}_{\text {left }}-\mathrm{RT}_{\text {right }}\right)$ was $\sim 74 \mathrm{~ms}$ higher in $\mathrm{RW}(72 \mathrm{~ms})$ than in controls $[-2 \pm 22 \mathrm{~ms}$, mean $\pm \mathrm{SD}$; one-tailed single-case $t$ test Crawford and Howell, 1998), $t=3.32, p=0.003$ ]. In rightward gaze, this index was $\sim 24 \mathrm{~ms}$ lower in RW $(-12 \mathrm{~ms})$ than in controls $(12 \pm 13 \mathrm{~ms}$; one-tailed single-case $t$ test, $t=1.78, p=$ $0.049)$. One-tailed $t$ tests were used because of the a priori hypothesis that visual sensitivity would increase nearer the midline after an S1 lesion (Odoj and Balslev, 2013). A repeated-measures ANOVA with factors direction of gaze (leftward or rightward) and group (RW or controls) implemented as an unstandardized difference test (Crawford et al., 1998; Crawford and Garthwaite, $2005)$ showed a significant interaction for the laterality index ( $p=0.001$, correlation between the difference in reaction times for the leftward and rightward gaze condition in the control group, $r=0.2$ ). Like in previous studies (Balslev et al., 2011b, their Fig. 3; Odoj and Balslev, 2013, their Fig. 2), an incidental observation was a rightward bias in healthy controls when the screen was placed to the right of midline (single-sample $t$ test, $t=$ 3.47, $p=0.004)$.

Discrimination accuracy for the control group over all conditions approached ceiling (screen left, $97.3 \pm 2.1 \%$; screen right, $96.9 \pm 2.8 \%)$ and was not significantly different compared with RW (95.3\% screen left and 97.9\% screen right, single-case comparisons, all $p>0.3)$.

In summary, Study 1 showed that an S1 lesion is associated with longer fixations at locations nearer midline during visual search (Experiment A) and an advantage for visual discrimination at these locations (Experiment B). These results suggest that an $\mathrm{S} 1$ lesion causes a bias in spatial attention that favors locations nearer the midline.

\section{Study 2: VBLM in right-hemisphere stroke patients}

Coinjury of S1 together with the neglect-typical perisylvian lesion is associated with a milder neglect

Patients with a right perisylvian stroke show contralesional neglect, namely a rightward bias in spatial attention (Karnath and Rorden, 2012). Notably, during visual search, neglect patients prefer to fixate away from the midline, in their right ipsilesional hemispace (Hornak, 1992; Karnath, 1994; Karnath et al., 1998). Because an isolated S1 lesion leads to a bias in spatial attention toward the midline, we hypothesized that, when S1 and the perisylvian network are injured together, the sum of the two effects may lead to a milder left-sided neglect. Study 2 investigated whether the coinjury of the postcentral gyrus in patients with a lesion of the perisylvian network reduces the rightward bias. This study was conducted in patients whose right hemisphere stroke overlapped at least $50 \%$ of the neglect-typical perisylvian region. Using VLBM, we searched for brain areas associated with a milder left neglect when lesioned rather than when intact. In accord with the hypothesis, an S1 lesion correlated with a lower neglect score. The area in the postcentral gyrus was the only cluster of voxels above the threshold (Fig. 6 A; threshold $p<0.05$, FDR corrected, $z$ score $>2.16$ ). Sixty-three percent of voxels were assigned to BA 1 (Fig. 6B) and 25\% to BA 2 (Fig. 6C) according to 
the Juelich atlas (Geyer et al., 2000; Grefkes et al., 2001; Eickhoff et al., 2005). Approximately $4 \%$ of the voxels were assigned to each of the areas $3 b, 6$, and IPC.

When we increased the sensitivity in a second VLBM analysis by excluding those voxels representing the perisylvian network region (and thus reducing the number of multiple comparisons), we again found only one cluster of significant voxels in the somatosensory cortex (threshold $p<0.05$, FDR corrected, $z$ score $>1.65)$. Sixty-two percent of these voxels were assigned to BA 1 and $32 \%$ to BA 2 according to the Juelich atlas.

Please note that the patients in the current analysis had a right hemisphere, predominantly cortical, lesion so the modulatory role of other brain areas known to alleviate left neglect when colesioned-such as the left frontal eye field (Vuilleumier et al., 1996), the left posterior parietal cortex (Brighina et al., 2003; Nyffeler et al., 2009), or superior colliculus (Sprague, 1966; Weddell, 2004)— could not be examined here.

The opposite comparison, which looked for brain areas associated with a more severe neglect when injured versus when intact, yielded no statistically significant voxels outside the perisylvian network region used to select the patients.

Neither lesion size nor the demographics can explain the lower $\mathrm{CoC}$ in the patients with S1 coinjury

Eight of the patients included in Study 2 had a lesion that fully or partially overlapped the somatosensory area identified by the VBLM analysis (Group 1), whereas in the remaining seven, the lesion did not cover this area (Group 2). As expected, Group 1 had a significantly milder neglect than Group 2 (mean \pm SD CoC, $0.48 \pm 0.37$ vs $0.85 \pm 0.09$, independent-samples $t$ test, $t=$ 2.6, $p=0.027)$. Although Group 1 had a lower neglect score, there was a numerical trend for the lesions in this group to overlap a larger percentage of the critical neglect area $(75 \pm 13 \%$ in Group 1 vs $62 \pm 12 \%$ in Group 2, $t=1.94, p=0.073$ ). The groups did not differ significantly in any of the other variables we measured, such as lesion size (13.2 $\pm 4.1 \mathrm{vs} 10.6 \pm 3.6 \mathrm{~cm}^{3}, t=$ $1.29, p>0.21)$, age ( $67 \pm 16$ vs $62 \pm 15$ years, $t=0.65, p>0.5)$, or time between the lesion and the neuropsychological testing ( $7 \pm 7$ vs $3 \pm 1.4 \mathrm{~d}, t=1.25, p>0.24)$.

Study 2 thus singled out the somatosensory cortex as the area associated with a milder left neglect when lesioned compared with when intact. These voxels were selected from a larger area in the cortex of the right hemisphere, located symmetrically around the neglect-typical lesion.

\section{Discussion}

An isolated S1 lesion caused a gradient in visual exploration as well as in visual sensitivity that favored locations nearer the midline. RW's bias in looking time despite normal oculomotor ability as well as the spatial asymmetry in her ability to discriminate retinally identical targets suggest that $\mathrm{S} 1$ plays a role in the allocation of attention in the body-centered space.

These results cannot be explained by an asymmetry in the motor response. First, Experiment A showed no significant difference between RW and controls in the range of eye movements or saccade metrics. RW's normal oculomotor range despite a lesion of the eye proprioceptive area is not surprising. The amplitude of visually guided saccades in both monkeys (Guthrie et al., 1983) and humans (Allin et al., 1996) was unimpaired when the eye proprioceptive input was removed or altered. A second and stronger argument than this null result is RW's spatial asymmetry in visual discrimination in Experiment B. In this experiment, participants fixated the gaze and responded using the same movements in all conditions. Therefore, a difference in reac- tion time between conditions cannot easily be attributed to an asymmetry in motor performance.

The current results do not reflect the patient's error in locating visual targets. Despite an erroneous proprioceptive signal from the left eye, this patient can, under normal circumstances, locate visual stimuli relative to the body as accurately and as precisely as healthy controls (Balslev et al., 2012a). This result is in line with the suggestion that, for locating visual objects relative to the body, the brain relies on the efference copy of the motor command rather than on eye proprioception (Lewis et al., 1998). Proprioceptive information is incorporated into the estimate of gaze only in special circumstances, when a mismatch with the efference copy is detected. Examples of such conditions are passive eye movement (Gauthier et al., 1990; Balslev et al., 2012a) or rTMS over the anterior parietal cortex (Balslev and Miall, 2008) but not a chronic S1 lesion (Balslev et al., 2012a).

A shift in visual exploration or visual sensitivity away from the periphery and toward the midline is not a general consequence of a right-hemispheric brain lesion. After such lesions, patients with or without spatial neglect have been tested on tasks similar to those in Experiment A (Karnath et al., 1998) and Experiment B (Smania et al., 1998). Neither of these studies found a bias toward the midline in spatial attention like that reported here. Instead, they observed a bias in the opposite direction, i.e., away from the midline and toward the ipsilesional periphery in neglect patients and the absence of a gradient in brain-damaged patients without neglect. This differs from the current findings and can thus rule out that a right-hemisphere lesion alone, regardless of its location, leads to a bias toward the midline in spatial attention.

Although the lesion in RW was limited to the postcentral gyrus, it is possible that a functional impairment that could not be detected in structural scans (i.e., a decrease in perfusion) could have reached farther into the surrounding areas. However, we are not aware of another brain area that, when lesioned, biases attention toward the midline. A function impairment in the posterior parietal or superior/middle temporal lobe-after a lesion (Karnath et al., 1996) or after an rTMS-induced "virtual lesion" (Nyffeler et al., 2008) — can bias visual exploration toward the contralesional space. This differs from the current findings. Likewise, we cannot exclude the possibility of a lesion of the connecting fibers between the intraparietal sulcus and the frontal eye field in RW. However, because these areas code saccade direction/ salient locations in the contralateral visual hemifield or contralateral body hemispace (Silver et al., 2005; Silver and Kastner, 2009; Szczepanski et al., 2010), it is unlikely that a lesion of these areas or their projection fibers could have caused the midlineperiphery gradient found here. Instead, this midline-periphery gradient fits well with the organization of the primary eye proprioceptive area. In monkeys, area 3a neurons increase their firing with ocular eccentricity for all directions of gaze (Wang et al., 2007). Assuming that the eye proprioceptive area in humans is organized in the same way, it is plausible that its lesion causes a gradient in attention from midline to periphery, in both ipsilesional and contralesional hemispace.

A lateral bias in visual exploration is a characteristic of patients with spatial neglect. These patients have a lesion centered on the sylvian sulcus, usually on the right side, which causes a shift in fixation time away from the midline and toward the ipsilesional hemispace (Hornak, 1992; Karnath, 1994; Karnath et al., 1998). The results of Study 2 showed that the extension of the neglect typical perisylvian lesion into $S 1$ reduces this lateral bias and thus leads to a milder neglect. This VLBM analysis included cortical voxels distributed symmetrically around the right sylvian sulcus 
to identify those whose lesion correlated with a reduced neglect severity. The only brain area identified by this analysis was located in S1. The subgroups of patients with and without an S1 coinjury did not differ significantly in lesion size, in the overlap between the lesion with the neglect-typical perisylvian area, in age, or in the time elapsed between stroke and neuropsychological testing. Thus, these variables cannot explain the milder neglect score in the subgroup of patients with S1 coinjury. The finding that an additional brain lesion can be an advantage for performance in neglect patients is counterintuitive. However, the shift in spatial selection toward midline after an isolated S1 lesion in Study 1 provides an explanation for the reduced lateral bias when the neglect-typical perisylvian lesion extends into this area.

We can think of two limitations in interpreting the current study. First, the current setup does not allow to tell whether this bias toward the midline after an S1 lesion occurred relative to a reference frame centered on the orbit, head, or trunk. This question can be addressed by further studies that dissociate head and trunk midline and present search arrays that are placed symmetrical relative to one of these body anchors.

Second, this study cannot answer where precisely S1 exerts its modulatory effect in the visual processing stream toward a motor response. Functional neuroimaging in patients with an S1 lesion would be needed to answer this question. In a previous study in which rTMS was applied to S1, we observed a modulation of the functional MRI activity in the cuneus (Balslev et al., 2012c). This variation in neural activity mirrors the center-to-periphery gradient in visual sensitivity observed here. These previous neuroimaging findings suggest that $\mathrm{S} 1$ could exert its modulatory activity on visual processing already at visual cortex level.

Spatial attention can be defined as the selection of locations for perception and/or for action (Bisley and Goldberg, 2010; Petersen and Posner, 2012). Within this framework, Studies 1 and 2 suggest a role of $\mathrm{S} 1 \mathrm{in}$ spatial attention. Given the neuroanatomi$\mathrm{cal}$ analogies between the oculomotor and spatial attention system (Corbetta, 1998), we hypothesize a coupling between the eye proprioceptive signal in S1 and spatial attention. One possibility is that the function of $S 1$ in spatial attention and in signaling the direction of gaze relies on independent neural populations that share the same principle of organization. For instance, this is the case in the frontal eye field, in which separate neural populations code the direction of a saccade and an attention shift (Juan et al., 2004; Thompson et al., 2005). A second possibility is that the locus of attention may be coded relative to the body by using a gaze-direction signal that has a proprioceptive component. We observed previously that direct manipulation of the extraocular muscles causes spatial gradients in visual attention (Balslev et al., 2012b). A role of eye proprioception in coding the locus of attention would be compatible with the delay in updating the locus of attention after an eye movement of $\sim 100-200 \mathrm{~ms}$ found in a previous study (Golomb et al., 2008). It would also be consistent with the latency of $150 \mathrm{~ms}$ until the gain field neurons in the lateral intraparietal area, which use eye position to implement non-retinotopic representations (Andersen et al., 1985), update their firing to reflect the new gaze direction after a saccade (Xu et al., 2012). These delays are in the same order of magnitude as the time needed by the eye proprioceptive signal to reach the cerebral cortex, $\sim 60 \mathrm{~ms}$ (Xu et al., 2011).

\section{Conclusion}

A lesion in the right $\mathrm{S} 1$, when it occurs in isolation, biases visual exploration and visual sensitivity toward the midline. When an
S1 lesion occurs as a part of a large perisylvian stroke, it is associated with a reduced bias toward the ipsilesional space and thus with a milder spatial neglect. These results argue for a novel role of the somatosensory cortex in visuospatial attention. We hypothesize that the proprioceptive gaze signal in S1, although playing only a minor role in locating visual objects relative to the body, affects the allocation of attention in the visual space.

\section{References}

Albert ML (1973) A simple test of visual neglect. Neurology 23:658-664. CrossRef Medline

Allin F, Velay JL, Bouquerel A (1996) Shift in saccadic direction induced in humans by proprioceptive manipulation: a comparison between memory-guided and visually guided saccades. Exp Brain Res 110:473481. Medline

Andersen RA, Essick GK, Siegel RM (1985) Encoding of spatial location by posterior parietal neurons. Science 230:456-458. CrossRef Medline

Balslev D, Miall RC (2008) Eye position representation in human anterior parietal cortex. J Neurosci 28:8968-8972. CrossRef Medline

Balslev D, Albert NB, Miall C (2011a) Eye muscle proprioception is represented bilaterally in the sensorimotor cortex. Hum Brain Mapp 32:624631. CrossRef Medline

Balslev D, Gowen E, Miall RC (2011b) Decreased visual attention further from the perceived direction of gaze for equidistant retinal targets. J Cogn Neurosci 23:661-669. CrossRef Medline

Balslev D, Himmelbach M, Karnath HO, Borchers S, Odoj B (2012a) Eye proprioception used for visual localization only in conflict with the oculomotor plan. J Neurosci 32:8569-8573. CrossRef Medline

Balslev D, Newman W, Knox PC (2012b) Extraocular muscle afferent signals modulate visual attention. Invest Ophthalmol Vis Sci 53:7004-7009. CrossRef Medline

Balslev D, Siebner HR, Paulson OB, Kassuba T (2012c) The cortical eye proprioceptive signal modulates neural activity in higher-order visual cortex as predicted by the variation in visual sensitivity. Neuroimage 61: 950-956. CrossRef Medline

Becker E, Karnath HO (2010) Neuroimaging of eye position reveals spatial neglect. Brain 133:909-914. CrossRef Medline

Bisley JW, Goldberg ME (2010) Attention, intention, and priority in the parietal lobe. Annu Rev Neurosci 33:1-21. CrossRef Medline

Borchers S, Hauser TK, Himmelbach M (2011) Bilateral hand representations in human primary proprioceptive areas. Neuropsychologia 49: 3383-3391. CrossRef Medline

Brett M, Leff AP, Rorden C, Ashburner J (2001) Spatial normalization of brain images with focal lesions using cost function masking. Neuroimage 14:486-500. CrossRef Medline

Brighina F, Bisiach E, Oliveri M, Piazza A, La Bua V, Daniele O, Fierro B (2003) $1 \mathrm{~Hz}$ repetitive transcranial magnetic stimulation of the unaffected hemisphere ameliorates contralesional visuospatial neglect in humans. Neurosci Lett 336:131-133. CrossRef Medline

Corbetta M (1998) Frontoparietal cortical networks for directing attention and the eye to visual locations: identical, independent, or overlapping neural systems? Proc Natl Acad Sci U S A 95:831-838. CrossRef Medline

Corbetta M, Shulman GL (2002) Control of goal-directed and stimulusdriven attention in the brain. Nat Neurosci Rev 3:201-215. CrossRef Medline

Crawford JR, Garthwaite PH (2005) Testing for suspected impairments and dissociations in single-case studies in neuropsychology: evaluation of alternatives using monte carlo simulations and revised tests for dissociations. Neuropsychology 19:318-331. CrossRef Medline

Crawford JR, Howell DC (1998) Comparing an individual's test score against norms derived from small samples. Clin Neuropsychol 12:482486. CrossRef

Crawford JR, Howell DC, Garthwaite PH (1998) Payne and Jones revisited: estimating the abnormality of test score differences using a modified paired samples t test. J Clin Exp Neuropsychol 20:898-905. CrossRef Medline

Duhamel JR, Colby CL, Goldberg ME (1992) The updating of the representation of visual space in parietal cortex by intended eye movements. Science 255:90-92. CrossRef Medline

Eickhoff SB, Stephan KE, Mohlberg H, Grefkes C, Fink GR, Amunts K, Zilles K (2005) A new SPM toolbox for combining probabilistic cytoarchitec- 
tonic maps and functional imaging data. Neuroimage 25:1325-1335. CrossRef Medline

Galletti C, Battaglini PP, Fattori P (1993) Parietal neurons encoding spatial locations in craniotopic coordinates. Exp Brain Res 96:221-229. Medline

Gauthier GM, Nommay D, Vercher JL (1990) The role of ocular muscle proprioception in visual localization of targets. Science 249:58-61. CrossRef Medline

Gauthier L, Dehaut F, Joannette I (1989) The bells test: a quantitative and qualitative test for visual neglect. Int J Clin Neuropsychol 11:49-54.

Geyer S, Ledberg A, Schleicher A, Kinomura S, Schormann T, Bürgel U, Klingberg T, Larsson J, Zilles K, Roland PE (1996) Two different areas within the primary motor cortex of man. Nature 382:805-807. CrossRef Medline

Geyer S, Schormann T, Mohlberg H, Zilles K (2000) Areas 3a, 3b, and 1 of human primary somatosensory cortex. Part 2 . Spatial normalization to standard anatomical space. Neuroimage 11:684-696. CrossRef Medline

Golomb JD, Chun MM, Mazer JA (2008) The native coordinate system of spatial attention is retinotopic. J Neurosci 28:10654-10662. CrossRef Medline

Grefkes C, Geyer S, Schormann T, Roland P, Zilles K (2001) Human somatosensory area 2: observer-independent cytoarchitectonic mapping, interindividual variability, and population map. Neuroimage 14:617631. CrossRef Medline

Guthrie BL, Porter JD, Sparks DL (1983) Corollary discharge provides accurate eye position information to the oculomotor system. Science 221: 1193-1195. CrossRef Medline

Hornak J (1992) Ocular exploration in the dark by patients with visual neglect. Neuropsychologia 30:547-552. CrossRef Medline

Juan CH, Shorter-Jacobi SM, Schall JD (2004) Dissociation of spatial attention and saccade preparation. Proc Natl Acad Sci U S A 101:15541-15544. CrossRef Medline

Karnath HO (1994) Spatial limitation of eye movements during ocular exploration of simple line drawings in neglect syndrome. Cortex 30:319330. CrossRef Medline

Karnath HO, Rorden C (2012) The anatomy of spatial neglect. Neuropsychologia 50:1010-1017. CrossRef Medline

Karnath HO, Fetter M, Dichgans J (1996) Ocular exploration of space as a function of neck proprioceptive and vestibular input-observations in normal subjects and patients with spatial neglect after parietal lesions. Exp Brain Res 109:333-342. Medline

Karnath HO, Niemeier M, Dichgans J (1998) Space exploration in neglect. Brain 121:2357-2367. CrossRef Medline

Karnath HO, Rennig J, Johannsen L, Rorden C (2011) The anatomy underlying acute versus chronic spatial neglect: a longitudinal study. Brain 134: 903-912. CrossRef Medline

Lewis RF, Gaymard BM, Tamargo RJ (1998) Efference copy provides the eye position information required for visually guided reaching. J Neurophysiol 80:1605-1608. Medline

Nyffeler T, Cazzoli D, Wurtz P, Lüthi M, von Wartburg R, Chaves S, Déruaz A, Hess CW, Müri RM (2008) Neglect-like visual exploration behaviour after theta burst transcranial magnetic stimulation of the right posterior parietal cortex. Eur J Neurosci 27:1809-1813. CrossRef Medline

Nyffeler T, Cazzoli D, Hess CW, Müri RM (2009) One session of repeated parietal theta burst stimulation trains induces long-lasting improvement of visual neglect. Stroke 40:2791-2796. CrossRef Medline

Odoj B, Balslev D (2013) Visual sensitivity shifts with perceived eye position. J Cogn Neurosci 25:1180-1189. CrossRef Medline

Petersen SE, Posner MI (2012) The attention system of the human brain: 20 years after. Annu Rev Neurosci 35:73-89. CrossRef Medline

Rorden C, Karnath HO, Bonilha L (2007) Improving lesion-symptom mapping. J Cogn Neurosci 19:1081-1088. CrossRef Medline

Rorden C, Karnath HO (2010) A simple measure of neglect severity. Neuropsychologia 48:2758-2763. CrossRef Medline

Silver MA, Kastner S (2009) Topographic maps in human frontal and parietal cortex. Trends Cogn Sci 13:488-495. CrossRef Medline

Silver MA, Ress D, Heeger DJ (2005) Topographic maps of visual spatial attention in human parietal cortex. J Neurophysiol 94:1358-1371. CrossRef Medline

Smania N, Martini MC, Gambina G, Tomelleri G, Palamara A, Natale E, Marzi CA (1998) The spatial distribution of visual attention in hemineglect and extinction patients. Brain 121:1759-1770. CrossRef Medline

Sprague JM (1966) Interaction of cortex and superior colliculus in mediation of visually guided behavior in the cat. Science 153:1544-1547. CrossRef Medline

Szczepanski SM, Konen CS, Kastner S (2010) Mechanisms of spatial attention control in frontal and parietal cortex. J Neurosci 30:148-160. CrossRef Medline

Thompson KG, Biscoe KL, Sato TR (2005) Neuronal basis of covert spatial attention in the frontal eye field. J Neurosci 25:9479-9487. CrossRef Medline

Vuilleumier P, Hester D, Assal G, Regli F (1996) Unilateral spatial neglect recovery after sequential strokes. Neurology 46:184-189. CrossRef Medline

Wang X, Zhang M, Cohen IS, Goldberg ME (2007) The proprioceptive representation of eye position in monkey primary somatosensory cortex. Nat Neurosci 10:640-646. CrossRef Medline

Weddell RA (2004) Subcortical modulation of spatial attention including evidence that the Sprague effect extends to man. Brain Cogn 55:497-506. CrossRef Medline

Weintraub S, Mesulam MM (1985) Mental state assessment of young and elderly adults in behavioral neurology. In: Principles of behavioral neurology, (Mesulam MM, ed), pp 71-123. Philadelphia: Davis Company.

Xu BY, Karachi C, Goldberg ME (2012) The postsaccadic unreliability of gain fields renders it unlikely that the motor system can use them to calculate target position in space. Neuron 76:1201-1209. CrossRef Medline

Xu Y, Wang X, Peck C, Goldberg ME (2011) The time course of the tonic oculomotor proprioceptive signal in area 3 a of somatosensory cortex. J Neurophysiol 106:71-77. CrossRef Medline 